\title{
Treatment patterns of antipsychotics and clinical features in patients with schizophrenia at the Psychiatric Ward of Tertiary hospital in Jakarta, Indonesia
}

\author{
Wawaimuli Arozal ${ }^{1}$, Diana Wijaya ${ }^{1}$, Vivian Soetikno ${ }^{1}$, Nurmiati Amir ${ }^{2}$ \\ ${ }^{I}$ Department of Pharmacology Faculty of Medicine Universitas Indonesia, Indonesia, ${ }^{2}$ Department of Psychiatry, \\ Faculty of Medicine Universitas Indonesia, Indonesia
}

Objective: Antipsychotics is a group of psychotropic drugs used as the mainstay treatment for schizophrenia and are used both to control acute relapses of symptoms and in maintenance therapy to reduce the risk of relapse. In Indonesia, studies on the pattern of drug use are still limited, particularly about the rational use of antipsychotics for schizophrenic patients. Irrational drug use will affect the effectiveness of the drug, as well as increase the incidence of side effects and drug interactions.

Methods: This retrospective study was conducted at the psychiatric ward of tertiary hospital in Jakarta, Indonesia. The medical records of the inpatients admitted over a 12-month period (July 2014 - June 2015) were collected from the beginning to the end of hospitalization. We collected the data including patient characteristics, clinical characteristics and the rational use of antipsychotics.

Results: In this study, of the 113 analyzed patients, the most commonly type of schizophrenia is paranoid, while the most commonly prescribed medication antipsychotics: were risperidone (63.7\%), haloperidol (20.4\%) and quetiapine (19.5\%). Surprisingly, we found that $73.4 \%$ patient met one or more criteria of inappropriateness treatment. Majority caused of inappropriateness were inappropriate indication (41.6\%) and using more than 1 antipsychotic $(37.2 \%)$.

Conclusion: Inpatients with schizophrenia disorder were exposed to a large number of inappropriate treatment. With the knowledge about medications the implementation of clinical pharmacology services to the psychiatrists would improve medication for inpatients with schizophrenia. 